\title{
FC++: Functional tools for object-oriented tasks*
}

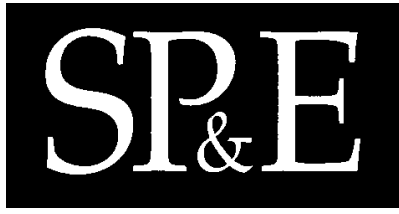

\author{
Yannis Smaragdakis and Brian McNamara ${ }^{\dagger}$ \\ College of Computing, Georgia Institute of Technology - Atlanta, GA 30332
}

\section{SUMMARY}

$\mathrm{FC}++$ is a library for programming functionally in $\mathrm{C}++$. Compared to other $\mathbf{C}++$ functional programming libraries, $\mathrm{FC}++$ is distinguished by its powerful type system which allows manipulating parametrically polymorphic functions (e.g., passing them as arguments to other functions and returning them as results).

In this paper, we show how $\mathrm{FC}++$ can be used in common OO programming tasks. We demonstrate $\mathrm{FC}++$ implementations of several common design patterns (Adapter, Builder, Command, and more). Compared to conventional $\mathrm{C}++$ implementations of these patterns, our implementations are either simpler (in that fewer classes/dependencies are needed), more efficient, or more type-safe (thanks to parametric polymorphism and type inference).

KEY WORDS: Design patterns, Object-oriented programming, Functional programming, C++, Parametric polymorphism

\section{Introduction}

Functional and object-oriented programming are the most active fields of research in programming languages and methodologies. Several pieces of work have attempted to connect the two paradigms. Among them are the Pizza language [1], extending Java, as well as libraries for programming functionally in $\mathrm{C}++[2,3,4,5] \mathrm{FC}++[6]$ is one such library, distinguished from all others by its powerful type system: $\mathrm{FC}++$ allows the programmer to define and fully manipulate parametrically polymorphic functions. The conventional $\mathrm{C}++$ way of representing polymorphic functions is via function templates, as in the $\mathrm{C}++$ Standard Library [7]. Nevertheless, function templates suffer severe limitations-e.g., they cannot be passed as parameters to other functions or returned as results. $\mathrm{FC}++$ polymorphic functions overcome these limitations, enabling $\mathrm{FC}++$ to re-implement straightforwardly many common

${ }^{*} \mathrm{FC}++$ web site: $\mathrm{http}: / / \mathrm{www} . \mathrm{cc} \cdot$ gatech.edu/ $\sim$ yannis/f c++/

$\dagger^{\dagger}$ Email: $\{$ yannis,lorgon\}@cc.gatech.edu 
functional operators (a large part of the Haskell Standard Prelude [8]). The library is currently quite rich in functionality and has an efficient implementation.

In a previous paper [6], we introduced $\mathrm{FC}++$ and its innovative type system, showed the components of the library, and demonstrated how its implementation is more efficient than previous similar attempts. In this paper we show how to leverage the library to create simple, efficient, and safe implementations of common OO design patterns [9].

Certainly a lot has been written about language support for implementing design patterns (e.g., $[10,11]$ ), functional techniques in OO programming, etc. Some of the approaches in the literature are even very close in philosophy to our work. For instance:

- Alexandrescu [12] demonstrates how the meta-programming capabilities of the $\mathrm{C}++$ language can be used to yield elegant pattern implementations.

- Kühne's dissertation proposes several patterns inspired by functional programming [13].

- Using functional techniques (higher-order functions) to implement the Observer and Command patterns is common - in fact, even standard practice in Java and Smalltalk.

- The benefits of polymorphic and higher-order functions have often been discussed in the functional programming literature [14].

Therefore, by necessity, part of our material presents a new mechanism but not new concepts. In particular, Section 3 shows that $\mathrm{FC}++$ offers a rich framework for OO tasks, but the pattern implementations shown are not novel: similar results can be obtained with other languages or libraries (although, among $\mathrm{C}++$ approaches, $\mathrm{FC}++$ is arguably the most complete).

Nevertheless, some advanced pattern implementations can use the more novel elements of $\mathrm{FC}++$ - mainly type inference and its ability to manipulate polymorphic functions. Such examples are presented in Section 4 and, to our knowledge, have not been discussed before. We show how parametric polymorphism can find its way into selected design patterns, how FC++ can handle such tasks, and how using parametric polymorphism results into more generic code. Note that we do not discuss improvements in design patterns' implementations by the mere addition of parametric typing (e.g., $\mathrm{C}++$ class templates) in a language. These are well understood and are even discussed in Reference [9], as implementation suggestions.

Although some $\mathrm{C}++$ background is required for much of the paper, we believe that the principles are interesting even to non-C ++ programmers. In particular, the paper offers insights for language designers and programmers by showing a platform where both subtype polymorphism and parametric polymorphism with type inference are readily available as complementary tools for problem solving.

\section{Background: Functional Programming with FC++}

We begin by briefly introducing the $\mathrm{FC}++$ library. For a more complete introduction to $\mathrm{FC}++$, the reader should refer to Reference [6].

FC++ Basics. In FC++, we express functions as instances of classes that follow certain conventions. We call such classes functoids. The key advantage to using functoids, rather than $\mathrm{C}++$ functions or function templates, is that we can pass them as parameters and return them as results - even if they are polymorphic. There are two kinds of functoids: direct and indirect. 
Direct functoids are the usual representation for functions in $\mathrm{FC}++$. Direct functoids can be either monomorphic or polymorphic. Indirect functoids, on the other hand, must always be monomorphic but can express first-class functions. That is, with indirect functoids, we can define variables that range over all functions with the same type signature. Thus, indirect functoids can be viewed as indirect function references, much like $\mathrm{C} / \mathrm{C}++$ function pointers. In addition to direct and indirect functoids, $\mathrm{FC}++$ provides a number of useful operations for creating functoids, composing them, specializing them, etc. We shall now discuss a few of the key components of $\mathrm{FC}++$ in more detail.

Indirect functoids are represented as the FunN family of classes. FunNs specify function signatures via template parameters; $\mathrm{N}$ is the number of arguments. For example, Fun2<int, char, string> is the type of a two-argument indirect functoid which takes an int and a char and returns a string. Note that the first $\mathrm{N}$ template arguments comprise the argument types of the function, and the last template argument is the result type. Thus, the simplest kind of indirect functoid is a Fun $0<$ void $>$ - a function that takes no arguments and returns no result.

A common way to create indirect functoids is with ptr_to_fun. ptr_to_fun transforms a normal $\mathrm{C}++$ function into a functoid. Here is a simple example, which also demonstrates how indirect functoids can range over different functions:

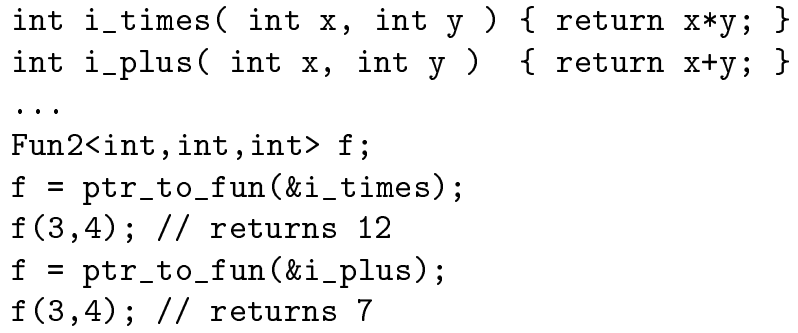

Note that $f$ 's behavior depends on which functoid it is bound to. This may seem reminiscent of OO dynamic dispatch (where a method call depends upon the dynamic type of the object that the receiver is bound to), and rightly so! There is just such a virtual method call buried inside the implementation of all indirect functoids.

Indirect functoids are more versatile than function pointers: they employ automatically currying, they can be bound to new function objects that are created on-the-fly, and they exhibit a form of subtype polymorphism (see [6] for details). What follows will demonstrate some of these features, which have important applications in Section 3 and Section 4.

Currying is a functional technique that allows us to bind a subset of a function's arguments to specific values. For example, we can use curry to bind the first argument of $f$ to the value 1 , creating a new one-argument function:

Fun1<int, int $>$ inc $=\operatorname{curry} 2(f, 1) ;$

inc(4); // returns 5 - i.e., i_plus $(1,4)$

(The 2 in curry 2 refers to the number of arguments that $f$ expects.) In fact, FC++ also allows the currying to be implicit - when a functoid is called with fewer actual arguments than 
it expects, it returns a curried functoid. For instance, the previous example can be written more simply as:

Fun $1<$ int, int> inc $=f(1)$;

inc (4); // returns 5 - i.e., i_plus $(1,4)$

Although this implicit form is what a typical $\mathrm{FC}++$ user would write, we will usually avoid it in this paper, in order to emphasize that currying is done through polymorphic functions (e.g., curry2) that manipulate other, possibly polymorphic, functions.

Functional composition is easily expressed with compose:

Fun1<int, int> inc2 = compose (inc, inc);

inc2(4); // returns 6 - i.e., inc(inc(4))

Currying and composition are among the powerful functional techniques for building new functions on-the-fly.

Unlike indirect functoids, direct functoids can be polymorphic. Consider the simple example of a function to create a std::pair. (std: :pair is the template struct in C++ used to represent a pair of values.) The direct functoid $\mathrm{mk}_{-}$pair makes a std: :pair from its two parameters. For example,

$$
\text { mk_pair (3, 'c') }
$$

returns a std: : pair structure whose first field is the int 3, and whose second field is the char ' $c$ '. Indeed, the $\mathrm{C}++$ standard defines a template function for the same purpose, which goes by the name std: :make_pair. However, compared to mk_pair, std: :make_pair suffers extreme limitations, by virtue of being defined as a template function. Template functions cannot be passed as parameters, which means we cannot use the functional techniques mentioned above (i.e., currying and composition) on templates. Direct functoids avoid these limitations. For example, we can say

curry2( mk_pair, 3 )

to return a new direct functoid which takes one argument of any type $\mathrm{T}$, and returns a std: : pair<int, $\mathrm{T}>$ whose first field is 3 . It is worth repeating that there is nothing special about the curry2 operator: it is just an $\mathrm{FC}++$ polymorphic direct functoid that manipulates other (possibly polymorphic) functoids. The ability to have higher-order polymorphic functions that manipulate other polymorphic functions is one of the features that sets $\mathrm{FC}++$ apart from other similar $\mathrm{C}++$ libraries.

Expressing Polymorphic FC++ Functoids. In the previous example, we demonstrated passing a polymorphic functoid to a higher-order functoid which returned a polymorphic result. How is this accomplished using $\mathrm{C}++$ ? The trick in $\mathrm{FC}++$ is to use a struct with nested template members for both the actual function and an explicit representation of the type signature of the functoid. The former is used so that we can exploit the language's inference of function argument types from the actual arguments to the function. The latter implements a type inference algorithm — given the input types to a function, compute the output type - using simple template computations. Thus in FC++ we would define mk_pair as: 


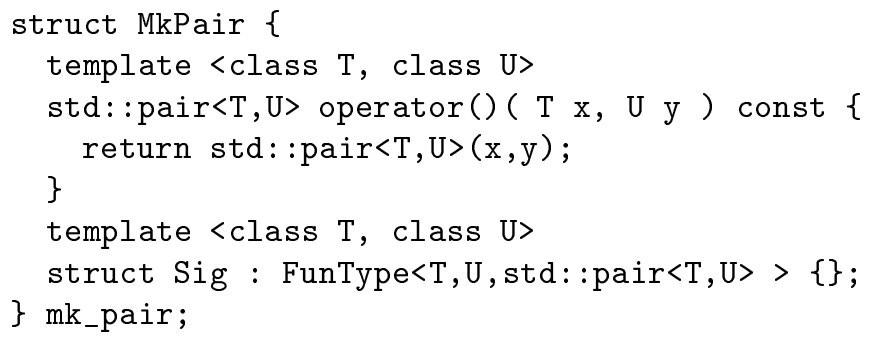

The operator () member (the usual way to define a function object in $\mathrm{C}++$ ) is defined just as we would expect. The key is the Sig member. FC++ functoids all have member structs named Sig which encode their function signatures. These Sigs contain typedefs named ResultType, First ArgType, etc., according to FC++ library conventions. To ease the task of defining such Sig members, we inherit the generic FunType class which defines the typedefs; FunType follows the same conventions as the indirect functoid FunN classes (the first few template parameters are the argument types and the final template parameter is the result type), but the template is specialized to accept a variable number of arguments (up to 7).

This encoding mechanism is the key that allows $\mathrm{FC}++$ to create higher-order functoids that can directly manipulate polymorphic functoids. Specifically, other functoids can determine what the result type of a particular polymorphic functoid would be, for given arguments.

To see how, consider the simple functoid apply, which applies a binary function to its arguments. That is, apply $(f, x, y)$ behaves just as $f(x, y)$ does. If $f$ is monomorphic, it is easy to implement such a function in $\mathrm{C}++$ using techniques from the STL [7]. However, suppose we want to use apply on a polymorphic function like mk_pair-how do we do it? In $\mathrm{FC}++$, we just say:

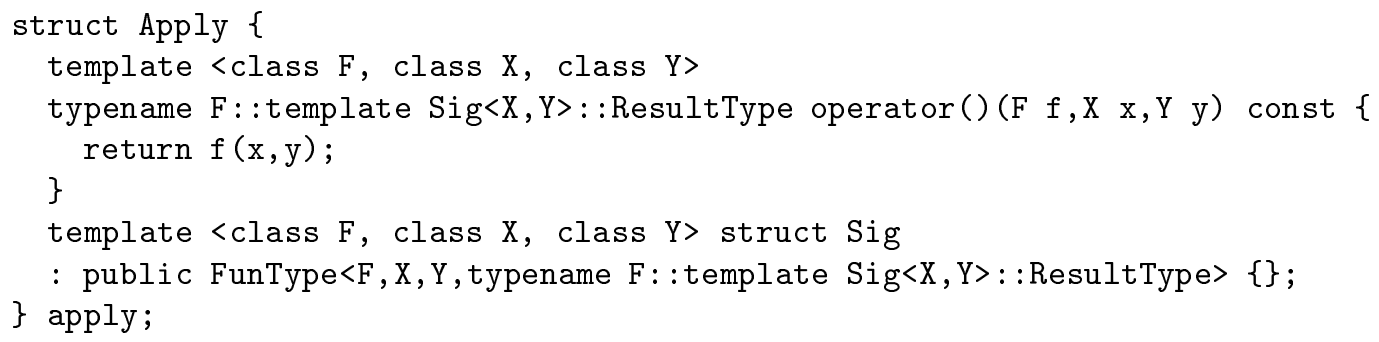

Note that apply's result type depends on both the type of the functoid and the types of arguments it receives;

$$
\text { F: : template } \mathrm{Sig}<\mathrm{X}, \mathrm{Y}>\text { : : ResultType }
$$

expresses this. Thus, for instance,

$\operatorname{apply}\left(\mathrm{mk}_{-}\right.$pair, 3, 'c')

returns a

MakePair: : Sig<int, char> : : ResultType 
which is just a typedef for

std : : pair<int, char>.

Note that apply also has its own nested Sig member, which means that apply itself could be manipulated by other higher-order functions.

The process of inferring a function's type from its arguments is called type inference. Type inference is automatic in modern functional languages (e.g., Haskell and ML). Type inference in $\mathrm{C}++$ is semi-automatic: the argument types can be inferred from the actual arguments, but there is no automatic way to infer the return type of a function. The Sig template member fills this role, providing a way to deduce the return type of a functoid based on its argument types.

As a more realistic example of type inference, consider the compose function applied to two unary functoids $f$ and $g$ of types $F$ and $G$, respectively. compose $(f, g)$ returns a (possibly polymorphic) direct functoid with the following Sig member:

template <class T>

struct Sig : public FunType<T, typename F: template Sig<

typename G: :template Sig<T>: :ResultType>: :ResultType> \{\} ;

That is (take a big breath): the return type of compose $(f, g)$ is a functoid of a single argument of type $T$, whose return type is the same as that of functoid $f$ when $f$ 's argument type is the same as the return type of functoid $g$ when g's argument is of type T.

Although the above examples may seem quite complicated, there are not too many useful higher-order functions like compose, and they are all already pre-defined in $\mathrm{FC}++$. As a result, clients are shielded from most of the complexity. Nevertheless, generic combinators like curry and compose owe their generality to the type inference mechanism. Thus, most of the FC++ examples we shall see in Section 4 are realizable only because of this unique feature of our library.

Despite FC++'s abilities, it is not a complete functional language with polymorphism and type inference. One of the main drawbacks is that variable types have to be declared explicitly. Although FC++ type inference eliminates the need for typing intermediate results, if the final result of an $\mathrm{FC}++$ expression needs to be stored, the variable must be explicitly typed.

\section{Reusability with Object-Oriented and Functional Patterns}

Functional programming promotes identifying pieces of functionality as just "functions" and manipulating them using higher-order operations on functions. These higher-order functions may be specific to the domain of the application or they may be quite general, like the currying and function composition operations are. Several design patterns [9] follow a similar approach through the use of subtype polymorphism. Subtype polymorphism allows code that operates on a certain class or interface to also work with specializations of the class or interface. This is analogous to higher-order functions: the holder of an object reference may express a generic algorithm which is specialized dynamically based on the value of the reference. Encapsulating 
functionality and data as an object is analogous to direct function manipulation. Other code can operate abstractly on the object's interface (e.g., to adapt it by creating a wrapper object).

It has long been identified that functional techniques can be used in the implementation of design patterns. For instance, the Visitor pattern is often considered a way to program functionally in OO languages. (The interested reader should see Reference [15] and its references for a discussion of Visitor.) The Smalltalk class MessageSend (and its variants, see Reference [16], p.254), the C++ Standard Library functors, Alexandrescu's framework (Reference [12], Ch. 5), etc., are all trying to capture the generic concept of a "function" and use it in the implementation of the Command or Observer pattern. In this section we will briefly review some of these well-known techniques, from the $\mathrm{FC}++$ standpoint, by using indirect functoids. In Section 4 we will consider how the unique features of $\mathrm{FC}++$ enable some novel implementations of other patterns.

Command. The Command pattern turns requests into objects, so that the requests can be passed, stored, queued, and processed by an object which knows nothing of either the action or the receiver of the action. An example application of the pattern is a menu widget. A pull-down menu, for instance, must "do something" when an option is clicked; Command embodies the "something". Command objects support a single method, usually called execute. Any state on which the method operates needs to be captured inside a command object.

The motivation for using the Command pattern is twofold. First, holders of command objects (e.g., menu widgets) are oblivious to the exact functionality of these objects. This decoupling makes the widgets reusable and configurable dynamically (e.g., to create context-sensitive graphical menus). Second, the commands themselves are decoupled from the application interface and can be reused in different situations (e.g., the same command can be executed from both a pull-down menu and a toolbar).

Here is a brief example which illustrates how Command might be employed in a wordprocessing application:

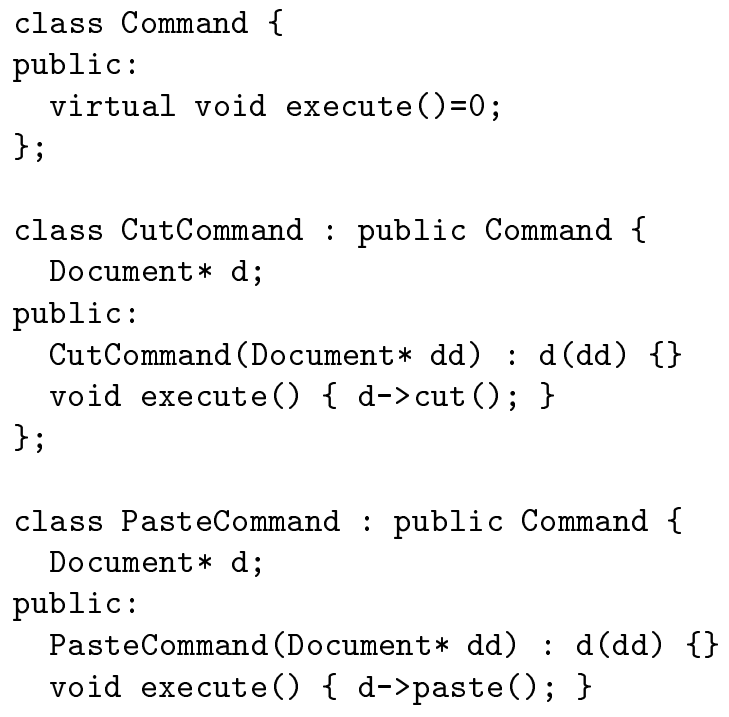




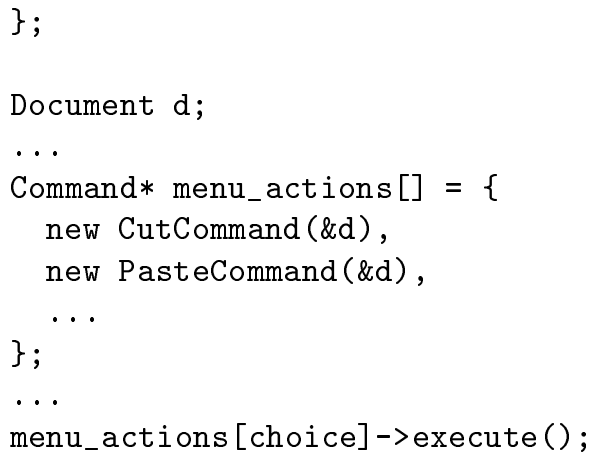

The abstract Command class exists only to define the interface for executing commands. Furthermore, the execute() interface is just a call with no arguments or results. In other words, the whole command pattern simply represents a "function object". From a functional programmer's perspective, Command is just a class wrapper for a "lambda" or "thunk" —an object-oriented counterpart of a functional idiom. Indirect functoids in $\mathrm{FC}++$ represent such function-objects naturally: a Fun0<void>can be used to obviate the need for both the abstract Command class and its concrete subclasses:

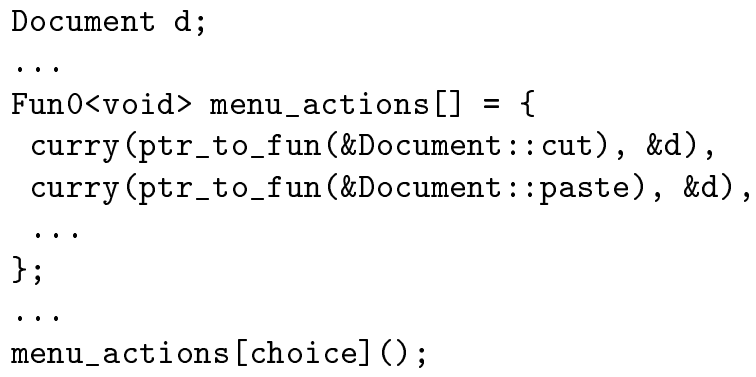

In this last code fragment, all of the classes that comprised the original design pattern implementation have disappeared! Fun $0<$ void $>$ defines a natural interface for commands, and the concrete instances can be created on-the-fly by making indirect functoids out of the appropriate functionality, currying arguments when necessary.

The previous example takes advantage of the fact that ptr_to_fun can be used to create functoids out of all kinds of function-like $\mathrm{C}++$ entities. This includes $\mathrm{C}++$ functions, instance methods (which are transformed into normal functions that take a pointer to the receiver

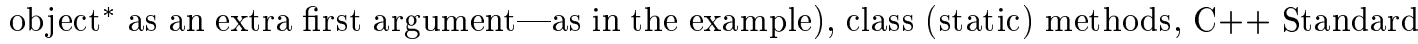
Library <functional> objects, etc. This is an example of design inspired by the functional paradigm: multiple distinct entities are unified as functions. The advantage of the unification is that all such entities can be manipulated using the same techniques, both application-specific and generic.

* Or a pointer to a const receiver object, if the method itself was const. The FC++ library strives to be const-correct. 
Observer. The Observer pattern is used to register related objects dynamically so that they can be notified when another object's state changes. The main participants of the pattern are a Subject and multiple Observers. Observers register with the subject by calling one of its methods (with the conventional name attach) and un-register similarly (via detach). The subject notifies observers of changes in its state, by calling an observer method (update).

The implementation of the observer pattern contains an abstract Observer class that all concrete observer classes inherit. This interface has only the update method, making it similar to just a single function, used as a callback. In fact, the implementation of the Observer pattern can be viewed as a special case of the Command pattern. Calling the execute method of the command object is analogous to calling the update method of an observer object.

The FC++ solution strategy for the Observer pattern is exactly the same as in Command. The Subject no longer cares about the type of its receivers (i.e., whether they are subtypes of an abstract Observer class). Instead, the interesting aspect of the receivers - their ability to receive updates - is encapsulated as a Fun $0<\mathrm{void}>$. The abstract Observer class disappears. The concrete observers simply register themselves with the subject. We will not show the complete code skeletons for the Observer pattern, as they are just specializations of the code for Command. Nevertheless, one aspect is worth emphasizing. Consider the code below for a concrete observer:

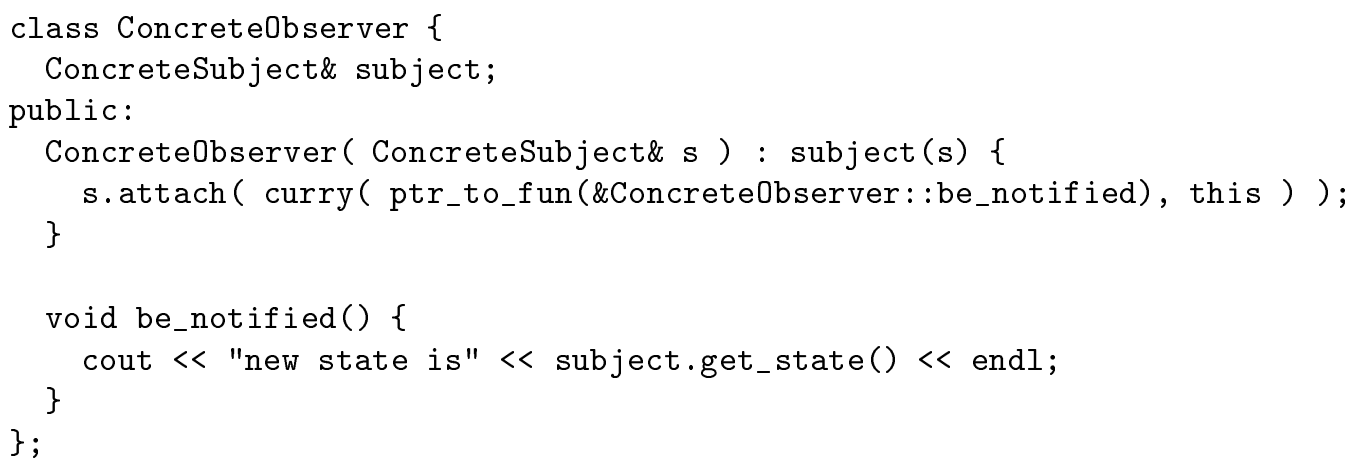

Note again how ptr_to_fun is used to create a direct functoid out of an instance method. The resulting functoid takes the receiver as its first parameter. curry is then used to bind this parameter. This approach frees observers from needing to conform to a particular interface. For instance, the above concrete observer implements be_notified instead of the standard update method, but it still works. Indeed, we can turn an arbitrary object into an observer simply by making a functoid out of one of its method calls - the object need not even be aware that it is participating in the pattern. This decoupling is achieved by capturing the natural abstraction of the domain: the function object.

Summarizing, the reason that Fun $0<\mathrm{void}>$ can replace the abstract Observer and Command classes is because these classes serve no purpose other than to create a common inteface to a function call. In Command, the method is named execute(), and in Observer, it is called update(), but the names of the methods and classes are really immaterial to the pattern. Indirect functoids in $\mathrm{FC}++$ remove the need for these classes, methods, and names, by instead 
representing the core of the interface: a function call which takes no argument and returns nothing.

C++'s parameterization mechanism lets us extend this notion to functions which take arguments and return values. For example, consider an observer-like scenario, where the notifier passes a value (for instance, a string) to the observer's update method, and the update returns a value (say, an integer). This can be solved using the same strategy as before, but using a Fun1<string, int> instead of a Fun0<void>. Again, the key is that the interface between the participants in the patterns is adequately represented by a single function signature ${ }^{\dagger}$; extra classes and methods (with fixed names) are unnecessary to realize a solution.

Virtual Proxy. The Virtual Proxy pattern seeks to put off expensive operations until they are actually needed. For example, a word-processor may load a document which contains a number of images. Since many of these images will reside on pages of the document that are off-screen, it is not necessary to actually load the entire image from disk and render it unless the user of the application actually scrolls to one of those pages. In [9], an ImageProxy class supports the same interface as an Image class, but postpones the work of loading the image data until someone actually requests it.

In many functional programming languages, the Virtual Proxy pattern is unnecessary. This is because many functional languages employ lazy evaluation. This means that values are never computed until they are actually used. This is in contrast to strict languages (like all mainstream OO languages), where values are automatically computed when they are created, regardless of whether or not they are used.

Since $\mathrm{C}++$ is strict, $\mathrm{FC}++$ is also strict by default. Nevertheless, a value of type $\mathrm{T}$ can be made lazy by wrapping the computation of that value in a Fun $0<\mathrm{T}>$. This is a common technique in strict functional languages. It encapsulates a computation as a function and causes the computation to occur only when the function is actually called (i.e., when the result is needed). For instance, in $\mathrm{FC}++$ a call $\mathrm{foo}(\mathrm{a}, \mathrm{b})$ can be delayed by writing it as curry2 $(f \circ o, a, b)$. The latter expression will return a 0 -argument functoid that will perform the original computation, but only when it is called. Thus, passing this functoid around enables the composition to be evaluated lazily.

We should mention that $\mathrm{FC}++$ defines some more tools for conveniently expressing lazy computations. First, the LazyPtrProxy class in FC++ works as a generic form of the ImageProxy mentioned earlier. A LazyPtrProxy has the same interface as a pointer to an object, but it does not actually create the object until it is dereferenced. That is, LazyPtrProxy is a way to delay object construction (as opposed to method calls). Second, FC++ contains an implementation of a lazy list data structure. This enables interesting solutions to some problems. For example, to compute the first $N$ prime numbers, we might create an infinite (lazy) list of all the primes, and then select just the first $N$ elements of that list. FC++ lazy lists are compatible with the data structures in the $\mathrm{C}++$ Standard Library and can be processed by a multitude of predefined $\mathrm{FC}++$ functions.

\footnotetext{
$\dagger$ A tuple of indirect functoids can be used if multiple function signatures are defined in an interface; the example in [9] of Command used for do/undo could be realized in FC++ with a std: : pair<Fun0<void>, Fun0<void>>, for instance.
} 


\section{Design Patterns and Parametric Polymorphism}

In the previous section, we saw how several common design patterns are related to functional programming patterns. All of our examples relied on the use of higher order functions. Another trait of modern functional languages (e.g., ML and Haskell) is support for parametric polymorphism with type inference. Type inference was discussed in Section 2: it is the process of deducing the return type of a function, given specific arguments. In this section, we will examine how some design pattern implementations can be improved if they employ parametric polymorphism with type inference and how they can further benefit from the entire arsenal of $\mathrm{FC}++$ techniques for manipulating these polymorphic functions.(The discussion of this section is only relevant for statically typed OO languages, like Java, Eiffel, or $\mathrm{C}++$. The novelties of $\mathrm{FC}++$ are in its type system - it has nothing new to offer to a dynamically typed language, like Smalltalk.)

Parametric vs. Subtype Polymorphism. Design patterns are based on subtype polymorphism - the cornerstone of OO programming. Parametric polymorphism, on the other hand, is not commonly available in OO languages, and even when it is, its power is limitede.g., there is no type inference capability. $\mathrm{FC}++$ adds this capability to $\mathrm{C}++$. It is interesting to ask when parametric polymorphism can be used in place of subtype polymorphism and what the benefits will be, especially in the context of design pattern implementations.

Parametric polymorphism is a static concept: it occurs entirely at compile time. Thus, to use a parametrically polymorphic operation, we need to know the types of its arguments at each invocation site of the operation (although the same operation can be used with many different types of arguments). In contrast, subtype polymorphism supports dynamic dispatch: the exact version of the executed operation depends on the run-time type of the object, which can be a subtype of its statically known type.

Therefore a necessary condition for employing parametric polymorphism is to statically know the type of operands of the polymorphic operation at each invocation site. When combined with type inference, parametric polymorphism can be as convenient to use as subtype polymorphism and can be advantageous for the following reasons:

- No common supertype is required. The issue of having an actual common superclass or just supporting the right method signature is similar to the named/structural subtyping dilemma. All mainstream OO languages except Smalltalk use named subtyping: a type A needs to declare that it is a subtype of B. In contrast, in structural subtyping, a type A can be a subtype of type B if it just implements the right method signatures. The advantage of requiring a common superclass is that accidental conformance is avoided. The disadvantage is that sometimes it is not easy (or even possible) to change the source code of a class to make it declare that it is a subtype of another. For instance, it may be impossible to modify pre-compiled code, or it may be tedious to manipulate existing inheritance hierarchies, or the commonalities cannot be isolated due to language restrictions (e.g., no multiple inheritance, no common interface signature). Even in languages like Java where a supertype of all types exists (the Object type), problems arise with higher-order polymorphic functions, like our curry operator. The problem is that an Object reference may be used to point to any object, but it cannot be passed to 
a function that expects a reference of a specific (but unknown) type. Thus, implementing a fully generic curry with subtype polymorphism is impossible.

- Type checking is static. With subtype polymorphism, errors can remain undetected until run-time. Such errors arise when an object is assumed to be of a certain dynamic type but is not. Since the compiler can only check the static types of objects, the error cannot be detected at compile-time. In fact, for many of the most powerful and general polymorphic operations, subtype polymorphism is impossible to use with any kind of type information. For instance, it would be impossible to implement a generic compose operator with subtype polymorphism, unless all functions composed are very weakly typed (e.g., functions from Objects to Objects). The same is true with most other higher-order polymorphic operations (i.e., functions that manipulate other functions).

- Method dispatch is static. Despite the many techniques developed for making dynamic dispatch more efficient, there is commonly a run-time performance cost, especially for hard-to-analyze languages like $\mathrm{C}++$. Apart from the direct cost of dynamic dispatch itself, there is also an indirect cost due to lost optimization opportunities (such as inlining). Therefore, when parametric polymorphism can be used in place of subtype polymorphism, the implementation typically becomes more efficient.

The examples that follow illustrate the advantages of using parametric polymorphism in the implementations of some design patterns.

Adapter. The Adapter pattern converts the interface of one class to that of another. The pattern is often useful when two separately developed class hierarchies follow the same design, but use different names for methods. For example, one window toolkit might display objects by calling paint (), while another calls draw (). Adapter provides a way to adapt the interface of one to meet the constraints of the other.

Adaptation is remarkably simple when a functional design is followed. Most useful kinds of method adaptation can be implemented using the currying and functoid composition operators of $\mathrm{FC}++$, without needing any special adapter classes. These adaptation operators are very general and reusable.

Consider the Command or Observer pattern. As we saw, in an FC++ implementation there is no need for abstract Observer or Command classes. More interestingly, the concrete observer or commands do not even need to support a common interface - their existing methods can be converted into functoids. Nevertheless, this requires that the existing methods have the right type signature. For instance, in our ConcreteObserver example, above, the be_notified method was used in place of a conventional update method, but both methods have the same signature: they take no arguments and return no results. What if an existing method has almost the right signature, or if methods need to be combined to produce the right signature?

For an example, consider a class, AnObserver, that defines a more general interface than what is expected. AnObserver may define a method:

void update(Time timestamp) $\{\ldots\}$

We would like to use this method to subscribe to some other object's service that will issue periodic updates. As shown in the Observer pattern implementation, the publisher expects a functoid object that takes no arguments. This is easy to effect by adapting the observer's interface: 
curry2( ptr_to_fun(\&AnObserver: :update), this, current_time())

In the above, we used a constant value (the current time) to specialize the update method so that it conforms to the required interface. That is, all update events will get the same timestamp-one that indicates the subscription time instead of the update time. A better approach is:

compose ( curry2 (ptr_to_fun(\&AnObserver: :update), this), ptr_to_fun (current_time))

In this example we combined currying with function composition in order to specialize the interface. The resulting function takes no arguments but uses global state (returned by the current_time() routine) as the value of the argument of the update method. In this way, each update will be correctly timestamped with the value of the system clock at the time of the update!

Other parametric polymorphism approaches (e.g., the functional part of the $\mathrm{C}++$ Standard Library [7], or Alexandrescu's framework for functions [12], Ch.5) support currying and composition for monomorphic functions. The previous examples demonstrate the value of type inference, which is not unique to $\mathrm{FC}++$. Nevertheless, $\mathrm{FC}++$ also extends type inference to polymorphic functions. We will see examples of currying and composition of polymorphic operations in the implementations of the next few patterns.

Decorator. The Decorator pattern is used to attach additional responsibilities to an object. Although this can happen dynamically, most of the common uses of the Decorator pattern can be handled statically. Consider, for instance, a generic library for the manipulation of windowing objects. This library may contain adapters, wrappers, and combinators of graphical objects. For example, one of its operations could take a window and annotate it with vertical scrollbars. The problem is that the generic library has no way of creating new objects for applications that may happen to use it. The generic code does not share an inheritance hierarchy with any particular application, so it is impossible to pass it concrete factory objects (as it cannot declare references to an abstract factory class).

This problem can be solved by making the generic operations be parametrically polymorphic and enabling type inference. For instance, we can write a generic $\mathrm{FC}++$ functoid that will annotate a window with a scrollbar:

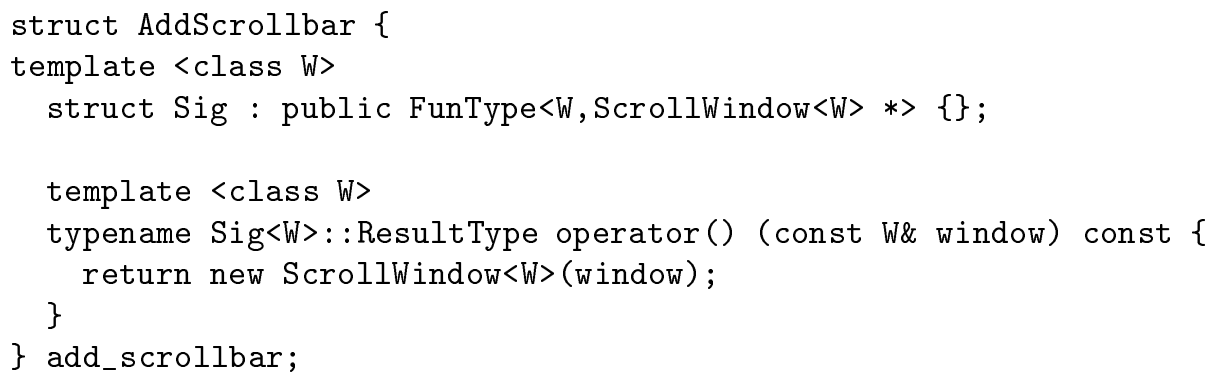

The above decorator functoid can be used with several different types of windows. For a window type $\mathrm{W}$, the functoid's return type will be a pointer to a decorated window type: ScrollWindow $\langle W\rangle$. (In fact, ScrollWindow can be a mixin, inheriting from its parameter, W.) 
Since the functoid conforms to the $\mathrm{FC}++$ conventions, it can be manipulated using the standard FC++ operators (e.g., composed with other functoids, curried, etc.). Composition is particularly useful, as it enables creating more complex generic manipulators from simple ones. For instance, a function to add both a scrollbar and a title bar to a window can be expressed as a composition:

compose(add_titlebar, add_scrollbar)

instead of adding a new function to the interface of a generic library. Similarly, if the add_titlebar operation accepts one more argument (the window title), the currying operation can be used (implicitly in the example below):

add_titlebar("Window Title")

The previous examples showed how classes can be statically decorated, possibly with new abilities added to them. Nevertheless, a common kind of decoration is pure wrapping, where the interface of the class does not change, but old operations are extended with extra functionality. Using parametric polymorphism one can write special-purpose polymorphic wrappers that are quite general. These could also be written as $\mathrm{C}++$ function templates, but if they are written as $\mathrm{FC}++$ functoids, they can be applied to polymorphic functoids and they can themselves be manipulated by other functoids (like curry and compose). Consider, for instance, an instrumentation functoid that calls a one-argument operation, prints the result of the invocation (regardless of its type) and returns that same result:

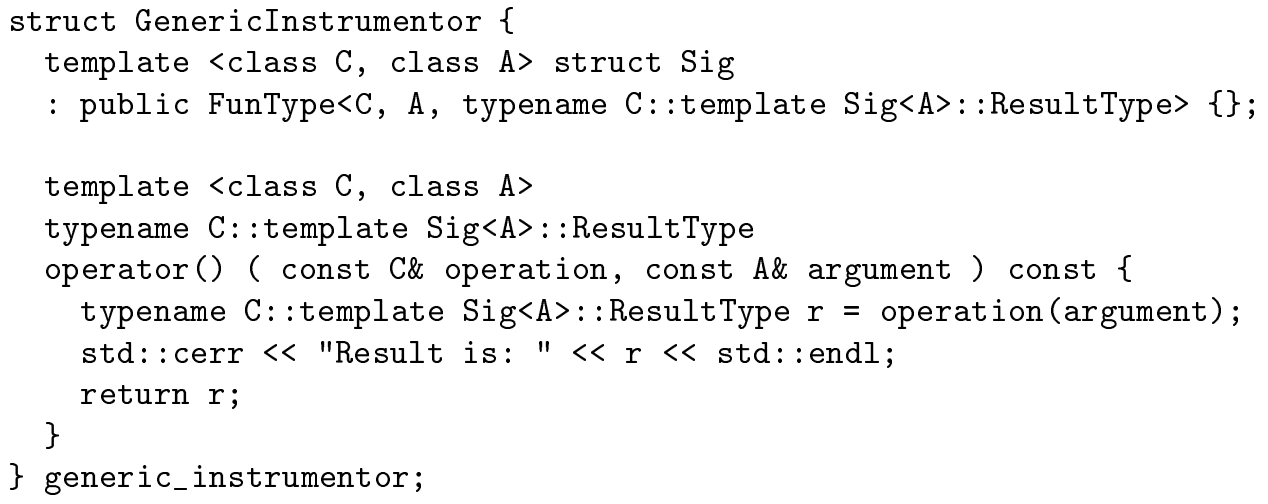

GenericInstrumentor exemplifies a special-purpose functoid (it logs the results of calls to an error stream) that can be generally applied (it can wrap any one-argument function).

Builder. The Builder design pattern generalizes the construction process of conceptually similar composite objects so that a generic process can be used to create the composite objects by repeatedly creating their parts. More concretely, the main roles in a Builder pattern are those of a Director and a Builder. The Director object holds a reference to an abstract Builder class and, thus, can be used with multiple concrete Builders. Whenever the Director needs to create a part of the composite object, it calls the Builder. The Builder is responsible for aggregating the parts to form the entire object. 
A common application domain for the Builder pattern is that of data interpretation. For instance, consider an interpreter for HTML data. The main structure of such an interpreter is the same, regardless of whether it is used to display web pages, to convert the HTML data into some other markup language or word-processing format, to extract the ASCII text from the data, etc. Thus, the interpreter can be the Director in a Builder pattern. Then it can call the appropriate builders for each kind of document element it encounters in the HTML data (e.g., font change, paragraph end, text strings, etc.).

In the Builder pattern, the Director object often implements a method of the form:

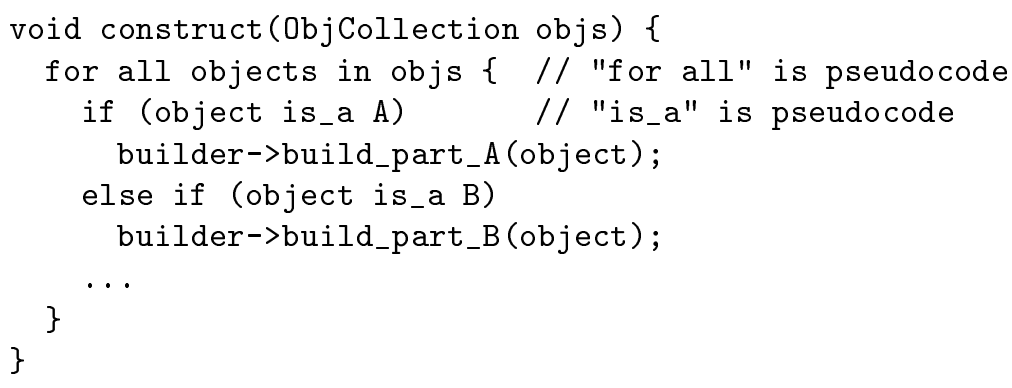

Note that the build_part method of the builder objects returns no result. Instead, the Builder object aggregates the results of each build_part operation and returns them through a method (we will call it get_result). This method is called by a client object (i.e., not the Director!).

A more natural organization would have the Director collect the products of building and return them to the client as a result of the construct call. In an extreme case, the get_result method could be unnecessary: the Director could keep all the state (i.e., the accumulated results of previous build_part calls) and the Builder could be stateless. Nevertheless, this is impossible in the original implementation of the pattern. The reason for keeping the state in the Builders is that Directors have no idea what the type of the result of the build_part method might be. Thus, Directors cannot declare any variables, containers, etc. based on the type of data returned by a Builder. Gamma et al. [9] write: "In the common case, the products produced by the concrete builders differ so greatly in their representation that there is little to gain from giving different products a common parent class."

This scenario (no common interface) is exactly one where parametric polymorphism is appropriate instead of subtype polymorphism. Using parametric polymorphism, the Director class could infer the result types of individual Builders and define state to keep their products. Of course, this requires that the kind of Builder object used (e.g., an HTML to PDF converter, an on-screen HTML browser, etc.) be fixed for each iteration of the construct loop, shown earlier. This is, however, exactly how the Builder pattern is used: the interpretation engine does not change in the middle of the interpretation. Thus, the pattern is static - another reason to prefer parametric polymorphism to subtyping. This may result in improved performance because the costs of dynamic dispatch are eliminated.

The new organization also has other benefits. First, the control flow of the pattern is simpler: the client never calls the Builder object directly. Instead of the get_result call, the results are returned by the construct call made to the Director. Second, Directors can now be more 
sophisticated: they can, for instance, declare temporary variables of the same type as the type of the Builder's product. These can be useful for caching previous products, without cooperation from the Builder classes. Additionally, Directors can now decide when the data should be consumed by the client. For instance, the Observer pattern could be used: clients of an HTML interpreter could register a callback object. The Director object (i.e., the interpreter) can then invoke the callback whenever data are to be consumed. Thus, the construct method may only be called once for an entire document, but the client could be getting data after each paragraph has been interpreted.

Another observation is that the Director class can be replaced by a functoid so that it can be manipulated using general tools. Note that the Director class in the Builder pattern only supports a single method call. Thus, it can easily be made into a functoid. Calling the functoid will be equivalent to calling construct in the original pattern. The return type of the functoid depends on the type of builder passed to it as an argument (instead of being void). An example functoid which integrates these ideas is shown here:

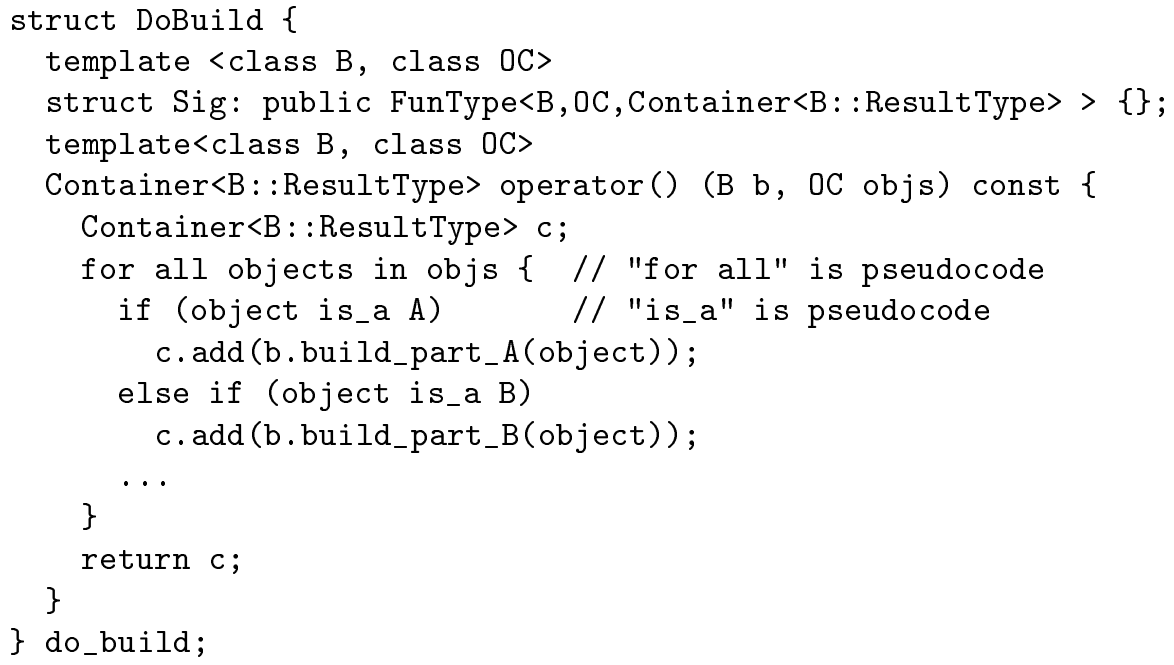

With this approach, the "director" functoid is in full control of the data production and consumption. The Director can be specialized via currying to be applied to specific objects or to use a specific Builder. Two different Directors can even be composed - the first building process can assemble a builder object for the second!

\section{Pragmatics}

In this section we briefly discuss some practical issues related to the $\mathrm{FC}++$ library.

Performance. The implementation of $\mathrm{FC}++$ imposes minimal overhead. Using direct functoids is as efficient as calling a $\mathrm{C}++$ function directly. Nevertheless, wrapping a native $\mathrm{C}++$ function into a direct functoid may prevent the compiler from uncovering opportunities for inlining. Similarly, creating functoids by currying and composition may introduce an extra 
function call. In both cases, however, the user has no more efficient option at the language level. For instance, if currying is required and the user does not resort to manual specialization (which could be done regardless of whether $\mathrm{FC}++$ is used) the overhead is unavoidable.

Indirect functoids suffer the cost of an extra indirection compared to direct function calls. Again, however, this cost is unavoidable if the user needs to refer to unknown functions through variables. Finally, $\mathrm{FC}++$ hides the details of reference management for indirect functoids by employing reference counting. In this way, indirect functoids can be created and used without need to be explicitly deallocated after the last reference to a functoid becomes unreachable. The reference counting mechanism introduces very small overhead. In previous work [6] we showed that using reference counting resulted in code faster by a factor of 4 to 8 compared to the "bridge" pattern used by Läufer [4]. More recently, a number of new optimizations were applied to the library implementation; reference [17] describes the details and quantifies the benefits. Nevertheless, the difference, as well as any overhead of reference counting, is very unlikely to appear in programming patterns like the ones described in this paper. Unless one uses data structures that create thousands of functoids (e.g., FC++ lazy lists), the overhead is non-existent.

Applications. $\mathrm{FC}++$ has already proven useful for functional programmers by providing an alternative, efficient platform for implementing familiar designs. An example of this approach is the XR (Exact Real) library [18]. XR uses the FC++ infrastructure to provide exact (or constructive) real-number arithmetic, using lazy evaluation.

Interface with STL. The FC++ library is designed to interface easily with the $\mathrm{C}++$ Standard Library. For example, the FC++ lazy List class supports iterators of the STL style, enabling easy conversion to and from STL data structures or other libraries that utilize the same iterator concepts. Also, FC++ provides routines to adapt STL-style "functors" into $\mathrm{FC}++$ functoids. Finally, the entire $\mathrm{FC}++$ library is wrapped in namespace $\mathrm{fcpp}$ to prevent name collisions with other libraries.

\section{Related Work}

We have referred to some related work throughout the previous sections. Here we selectively discuss related work that we did not get the chance to analyze earlier.

There are several libraries that add functional programming features to $\mathrm{C}++$. Some of them $[2,3,5]$ focus on front-end support (e.g., a lambda keyword) for creating functions on-the-fly. Other libraries $[4,7]$ provide reusable functionality without any special front-end support. $\mathrm{FC}++[6]$ is in this latter category: it provides mechanisms for expressing higher order and polymorphic functions, but does not hide the implementation behind a more convenient front end. $\mathrm{FC}++$ is distinguished from the rest by its full type system for polymorphic functions, which enables creating and manipulating polymorphic functions on-the-fly, and by its support for indirect function references.

Dami's currying mechanism for $\mathrm{C} / \mathrm{C}++[19]$ was used to demonstrate the advantages of function specialization, but required a language extension. As we saw, the same benefits can be obtained in $\mathrm{C}++$ without extending the language. 
Alexandrescu [12] offers a mature $\mathrm{C}++$ implementation of the Abstract Factory pattern. His approach consists of a generic (i.e., polymorphic) Abstract Factory class that gets parameterized statically by all the possible products. It is worth noting that this is the exact scenario that Baumgartner et al. [10] studied. Their conclusion was that meta-object protocols should be added to OO languages for better pattern support. Thus, Alexandrescu's implementation is a great demonstration of the meta-programming capabilities of $\mathrm{C}++$ - the language's ability to perform template computation on static properties can often be used instead of meta-object protocols.

Géraud and Duret-Lutz [20] offer some arguments for redesigning patterns to employ parametric polymorphism. Thus, they propose that parametric polymorphism be part of the "language" used to specify patterns. In contrast, our approach is to use parametric polymorphism with type inference in the implementation of patterns. From an implementation standpoint, the Géraud and Duret-Lutz suggestions are not novel: they have long been used in $\mathrm{C}++$ design pattern implementations. Furthermore, the examples we offer in this paper are more advanced, employing type inference and manipulation of polymorphic functions.

The Pizza language [1] integrates functional-like support to Java. This support includes higher-order functions, parametric polymorphism, datatype definition through patterns, and more. Pizza operates as a language extension and requires a pre-compiler. Support for parametric polymorphism in Java has been a very active research topic (e.g., [21, 22, 23, 24]), and a solution based on GJ [22] has been recently adopted [25]. Type inference is used in GJ. Nevertheless, due to the GJ translation technique (erasure) it is not possible to extract static type information nested inside template parameters. Thus, it is not possible to use the GJ type system to pass polymorphic functions as arguments and return them as results (in a type-safe way) as we do in $\mathrm{FC}++$.

It should be noted that Java inner classes [26] are excellent for implementing higher-order functions. Inner classes can access the state of their enclosing class, and, thus, can be used to express closures - automatic encapsulations of a function together with the data it acts on. Java inner classes can be anonymous, allowing them to express anonymous functions - a capability that is not straightforward to emulate in $\mathrm{C}++$. Many of our observations of Section 3 also apply to Java. In fact, the most common Java implementations of the Command and Observer design patterns use inner classes for the commands/callbacks.

\section{Conclusions}

In this paper we examined how functional techniques in general, and $\mathrm{FC}++$ in particular, can be applied to OO tasks, by illustrating the implementations of some common design patterns. Our examples from Section 3 are similar to others in the literature, but, to our knowledge, our example pattern implementations from Section 4 have not appeared before, even in different contexts. Additionally, we are not aware of another mainstream, statically-typed OO language with the capabilities of $\mathrm{FC}++$ for manipulating polymorphic functions and employing type inference.

Our implementations demonstrate the value of parametric polymorphism and type inference (even in a rather primitive form) in a statically-typed object-oriented language. By selectively 
using parametric polymorphism with type inference and higher-order functions, we can create simple, yet general, implementations of patterns that are both efficient and type safe.

\section{ACKNOWLEDGEMENTS}

The authors were partially supported by the Yamacraw Foundation and DARPA/ITO under the PCES program.

\section{REFERENCES}

1. M. Odersky and P. Wadler, "Pizza into Java: Translating theory into practice", ACM Symposium on Principles of Programming Languages, 1997 (PoPL 97).

2. J. Järvi and G. Powell, "The Lambda Library: Lambda abstraction in C++", TUCS Tech. Report No. 378, November 2000, available from http://www.tucs.abo.fi/publications/techreports/TR378.html .

3. O. Kiselyov, "Functional style in $\mathrm{C}++$ : closures, late binding, and lambda abstractions", poster presentation, Int. Conf. on Functional Programming, 1998.

4. K. Läufer, "A framework for higher-order functions in C++", Proc. Conf. Object-Oriented Technologies (COOTS), Monterey, CA, June 1995.

5. J. Striegnitz, "FACT!-The functional side of C++", http://www.fz-juelich.de/zam/FACT

6. B. McNamara and Y. Smaragdakis, "FC++: Functional programming in $\mathrm{C}++$ ", Proc. International Conference on Functional Programming (ICFP), Montreal, Canada, September 2000.

7. A. Stepanov and M. Lee, "The Standard Template Library", 1995. Incorporated in ANSI/ISO Committee $\mathrm{C}++$ Standard

8. S. Peyton Jones and J. Hughes (eds.), Report on the Programming Language Haskell 98, available from www.haskell.org, February 1999.

9. E. Gamma, R. Helm, R. Johnson, and J. Vlissides, Design Patterns: Elements of Reusable Object-Oriented Software. Addison-Wesley, 1994.

10. G. Baumgartner, K. Läufer, V.F. Russo, "On the interaction of object-oriented design patterns and programming languages", Tech. Report CSD-TR-96-020, Dept. of Comp. Sci., Purdue University, February 1996.

11. C. Chambers, B. Harrison, and J. Vlissides, "A debate on language and tool support for design patterns", ACM Symposium on Principles of Programming Languages, 2000 (PoPL 00).

12. A. Alexandrescu, Modern C ++ Design: Generic Programming and Design Patterns Applied, AddisonWesley Professional, 2001

13. T. Kühne, A Functional Pattern System for Object-Oriented Design, Verlag Dr. Kovac, Hamburg, 1999.

14. S. Thompson, "Higher-order + polymorphic = reusable", unpublished, May 1997. Available at: http://www.cs.ukc.ac.uk/pubs/1997/224.

15. S. Krishnamurthi, M. Felleisen, D. P. Friedman, "Synthesizing object-oriented and functional design to promote re-use", European Conference on Object-Oriented Programming (ECOOP), Brussels, Belgium, July 1998

16. S.R. Alpert, K. Brown, B. Woolf, The Design Patterns Smalltalk Companion, Addison-Wesley, 1998.

17. B. McNamara and Y. Smaragdakis, "Functional programming with the FC++ library", Proc. of Second Workshop on C++ Template Programming, Tampa, FL, October 2001. Available at http://www . oonumerics.org/tmpw01/.

18. K. Briggs and Y. Smaragdakis, The XR Exact Real Home Page. http://www.btexact.com/people/briggsk2/XR.html .

19. L. Dami, "More functional reusability in $\mathrm{C} / \mathrm{C}++$ /Objective-C with curried functions", Object Composition, Centre Universitaire d'Informatique, University of Geneva, pp. 85-98, June 1991.

20. T. Géraud and A. Duret-Lutz, "Generic programming redesign of patterns", in Proc. European Conf. on Pattern Languages of Programs, 2000 (EuroPLoP'2000).

21. O. Agesen, S. Freund, and J. Mitchell, "Adding type parameterization to the java language", Proc. ObjectOriented Programming Systems, Languages, and Applications (OOPSLA) 1997, 49-65. 
22. G. Bracha, M. Odersky, D. Stoutamire and P. Wadler, "Making the future safe for the past: Adding genericity to the Java programming language", Proc. Object-Oriented Programming Systems, Languages, and Applications (OOPSLA) 1998.

23. A. Myers, J. Bank and B. Liskov, "Parameterized types for Java", ACM Symposium on Principles of Programming Languages, 1997 (PoPL 97).

24. K. Thorup, "Genericity in Java with virtual types", European Conference on Object-Oriented Programming (ECOOP) 1997, 444-471.

25. Gilad Bracha, "Add generic types to the Java programming language", Java Specification Request (JSR) 14, Sun Microsystems, 1999.

26. Javasoft, Java Inner Classes Specification, 1997. In http://java.sun.com/products/jdk/1.1/docs/ . 